\title{
Mycotic aneurysm as a complication of infective endocarditis - a case report
}

\section{ANEURISMA MICÓTICO COMO COMPLICAÇÃO DE ENDOCARDITE INFECCIOSA - RELATO DE CASO}

Maria Gabriela Lang ${ }^{1 *}$, Marielle Lang $^{2}$, Rafael Ronson $^{3}$

${ }^{1}$ Resident physician of Clinical Medicine at the Hospital Municipal São José, Joinville, SC, Brazil.

${ }^{2}$ Resident physician of Clinical Medicine at the Hospital Universitário da Região Norte do Paraná - State University of Londrina. Londrina, PR, Brazil.

${ }^{3}$ Assistant physician to the Clinical Medicine service at the Hospital Municipal São José - Joinville, SC, Brazil.

Study conducted at the Hospital Municipal São José, Joinville, SC. Approved by the Research Ethics Committee at the Hospital Municipal São José on 09/19/2013.

\section{INTRODUCTION}

The first mention of the clinical aspects of infectious endocarditis (IE) comes from French physician Jean François Fernel in the $16^{\text {th }}$ century. ${ }^{1}$ However, appropriate treatment only started being implemented more than a century later, when the presence of microorganisms as the cause of the disease was identified. ${ }^{2}$ IE normally affects the native valves as a primary site, being the mitral valve the most common. ${ }^{3}$ In the last thirty years there have been important advancements in the diagnostic and therapeutic methods for the disease, which despite the low incidence in the population in general, still presents a poor prognosis and high mortality rate. ${ }^{4,5}, \mathrm{IE}$ complications appear in $60 \%$ of patients, being mycotic aneurysm one of the most serious. Caused by weakening of the vessel walls, it is clinically recognized in $3-10 \%$ of IE cases. ${ }^{7}$ The most dramatic event is subarachnoid hemorrhage, which usually occurs in the stage of bacteremia. ${ }^{6}$

\section{Case report}

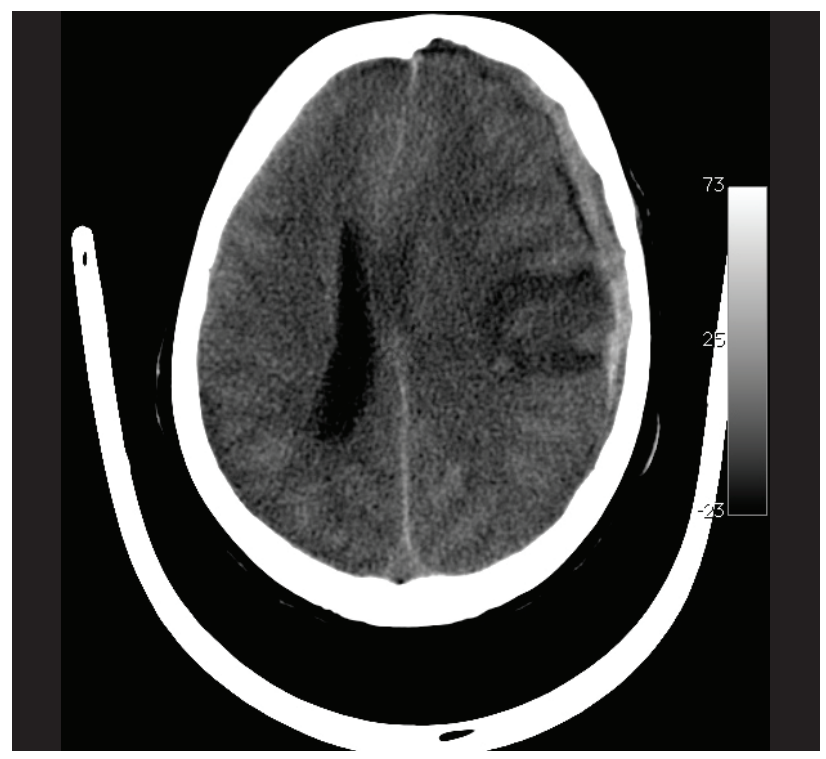

FIGURE 1 Computerized tomography of the cranium showing acute frontoparietal subdural hematoma to the left with a subjacent frontoparietal focus of contusion

Source: HMSJ neuroradiology service. 


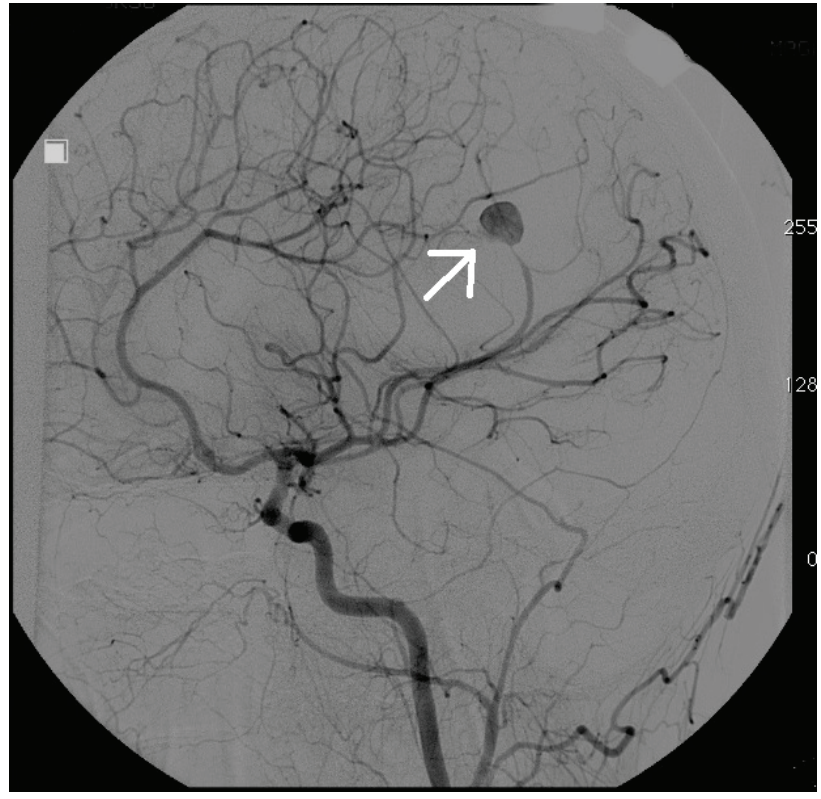

FIGURE 2 Angiography showing aneurysm formation, measuring about $8 \times 9 \mathrm{~mm}$ on topographic examination of cortical branch of the middle cerebral artery associated with parietal irregularity of the vessel, as indicated by the white arrow.

Source: HMSJ neuroradiology service.

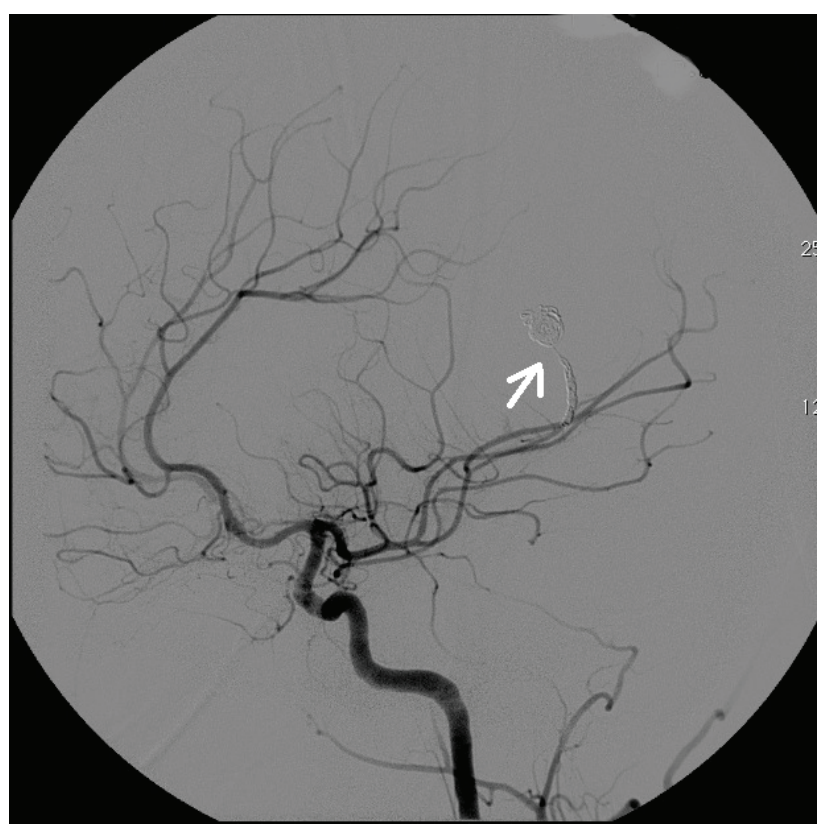

FIGURE 3 Postembolization arteriogram with coils, as indicated by the white arrow.

Source: HMSJ neuroradiology service.
Female patient, 45 years old, born in São Jorge d'Oeste, state of Paraná, Brazil, and originating from Joinville, Santa Catarina, white, married, building janitor. She was admitted to the Hospital Municipal São José (HMSJ) for investigation of epigastric pain associated with holocranial headache, intermittent and progressive, with 1 month of evolution and significant deterioration in the last 2 days. No further complaints.

Hypertensive and hypothyroid patient, treated with captopril $25 \mathrm{mg}$ T.I.D. and levothyroxine $50 \mathrm{mcg} /$ day. Underwent thyroidectomy 8 months before admission for adenomatous goiter. Patient denies allergies and previous hospitalization.

On physical examination, she showed good general condition, pale $+/ 4+$, hydrated, eupneic, tachycardia (HR: 110), afebrile, acyanotic, anicteric, lucid and oriented in time and space. In the lung auscultation, vesicular murmur present and symmetrical, with no adventitious sounds. In the cardiovascular examination, presence of systolic murmur from regurgitation, pancardiac $4+/ 6+$ radiating to the left armpit and more audible in the mitral zone, with tremor present, ictus cordis of normal size, palpable and visible. The presence of lesions compatible with Janeway lesions on the right fifth toe was verified. No signs of meningeal irritation and no focal deficits.

Additional tests were requested: cranial tomography that showed no presence of acute lesions; CSF without changes in cellularity, protein, glucose and culture-negative; negative blood culture; anti-HCV non-reactive, anti-HIV nonreactive and HBsAg reactive serologies; echocardiogram demonstrating redundant mitral valve with intense systolic posterior movement and nodule image on atrial side of the anterior leaflet suggesting vegetation.

Given the findings, IE diagnosis was established based on the modified Duke criteria (two major and one minor). ${ }^{8}$ Treatment started with $60 \mathrm{mg}$ of gentamicin T.I.D. and $1 \mathrm{~g}$ of vancomycin B.I.D. after hospital admission. Transesophageal ultrasound performed on the twentyseventh day of hospitalization showed a $40 \%$ reduction of mitral valve vegetation, with antibiotic treatment replaced by 2 g oxacillin Q.I.D.

On the fifty-seventh day of hospitalization, the patient had an episode of generalized tonic-clonic seizures associated with decreased level of consciousness - with no eye opening, issuing incomprehensible sounds and decorticate response to painful stimuli (Glasgow 06). Orotracheal intubation was then implemented. In cranial tomography performed after the event, the presence of acute subdural hematoma was found on the frontoparietal subdural hematoma to the left with a subjacent frontoparie- 
tal focus of contusion. The patient was sent for an emergency decompressive hemicraniectomy. The next day the patient was submitted to angiography, which revealed an aneurysm measuring about $8 \times 9 \mathrm{~mm}$ on topography of the cortical branch of the left middle cerebral artery associated with parietal irregularity of the vessel - image compatible with a ruptured mycotic aneurysm, which was embolized with coils. After the procedure, the patient was referred to the Intensive Care Unit at the hospital.

On the sixty-seventh day of hospitalization orotracheal extubation was performed, and after three days the patient was then referred to clinical medicine ward. The patient initially remained aphasic, but presented progressive improvement with multi-professional rehabilitation. The patient was discharged on the ninety-fifth day of hospitalization with moderate disability (grade 3 on the modified Rankin scale), ${ }^{9}$ after completing 42 days of treatment with the latest antimicrobial scheme. They were referred for monitoring by hepatitis and cardiology outpatient clinics (with valvuloplasty scheduled). The patient underwent skull reconstruction 60 days after discharge, with no adverse events.

\section{Discussion}

The case reported is consistent with the reality of IE in medical practice in various aspects. The patient, aged 47, and with native valves is categorized under the new epidemiological profile of the disease, which was previously reserved for patients with compromised valves and young adults. However, the entry point that could have caused bacteremia was not identified, as is possible in the majority of cases. ${ }^{10}$

The patient sought the health service with specific complaints, presenting a physical exam with various signs of cardiovascular impairment. This paradox is not frequent, but possible, considering the presence of underlying heart disease and the characteristics of the patient. ${ }^{6}$ The literature demonstrates that although approximately $90 \%$ of patients present fever and $80 \%$ have heart murmurs, the diagnosis is difficult. ${ }^{10}$ In the majority of cases, IE is only diagnosed accurately around one month after hospital admission, with transesophageal ultrasound as an indispensable exam for it presents sensitivity over $90 \%$. In the present case, the exam was capable of showing mitral valve vegetation, at the location most commonly affected by IE. ${ }^{11,12}$ Delays of weeks or months in antimicrobial treatment increase the chance of the occurrence of embolic or immunological events. ${ }^{6}$

In relation to the blood culture, the case presented differs from European studies where 2.5 to $31 \%$ of cases demonstrate negativity, but is very similar to that found in a Senegalese study conducted from 2005 to 2011, in which $60 \%$ of blood cultures were negative., ${ }^{42,13}$

After almost two months of clinical evolution the patient presented a mycotic aneurysm, as a consequence of displacement of septic emboli from valvular vegetation to the arterial vasa vasorum, disseminating the infection to the inner layer and wall of the vessel. ${ }^{5,13}$ This complication is rare, occurring in around $2-4 \%$ of cases, and as reported, more than half $(57.4 \%)$ are located in the distal branches of the medium cerebral artery.,13,14,15

The cerebral hemorrhage presented by the patient can be justified by the fact that mycotic aneurysms generally present a fine and fragile wall. ${ }^{7}$ A multicenter study conducted in France showed that $65-80 \%$ of mycotic aneurysms are asymptomatic, diagnosed only by imaging exams. In the case of rupture, however - as in the case presented here, in which the patient experienced convulsions and changes to her level of consciousness -, mortality reaches $80 \% .{ }^{5,10,11,14,16}$ In contrast with ruptured aneurysms, unruptured ones have a much lower mortality rate (30\%) and treatment is generally based on antibiotics and patient monitoring. ${ }^{5,14}$

In addition to the difficulty in diagnosis, the treatment of this complication does not yet have defined protocols, which makes conduct individualized and based on the clinical symptoms of the patient, as well as on the cost-benefit analysis of the treatment strategies. ${ }^{10}$ The majority of the Guidelines advise waiting 6 to 8 weeks for performing cardiac surgery on patients with a hemorrhagic event secondary to IE. ${ }^{15}$ Even if single antibiotic treatment is responsible for curing the majority of cases, not conducting surgery could lead to rupture of the aneurysm and catastrophic consequences. ${ }^{5}$

The long period of hospitalization of the patient (nearly 100 days) may be explained by the occurrence of cerebral hemorrhage, corroborated by the study conducted in northeastern Italy, which demonstrates an increase in the average hospitalization from 23 to 35 days in the presence of complications associated with IE. ${ }^{17}$ Despite this, like the majority of survivors of cerebrovascular events secondary to IE evaluated by the study by Yeates, the patient recovered well from her neurological impairments. ${ }^{15}$

\section{References}

1. Millar BC, Moore JE. Emerging issues in infective endocarditis. Emerg Infect Dis. 2004;10:1110-6.

2. Contrepois A. Towards a history of infective endocarditis. Med Hist. 1996;40:25-54

3. Netzer ROM, Altwegg SC, Zollinger E, Täuber M, Carrel T, Seiler C. Infective endocarditis: determinants of long term outcome. Heart. 2002;88:61-6. 
4. Leone S, Ravasio V, Durante-Mangoni E, Crapis M, Carosi G, Scotton PG, et al. Epidemiology, characteristics, and outcome of infective endocarditis in Italy: Italian study on endocarditis. Infection. 2012;40:527-35.

5. Bayer AS, Bolger AF, Taubert KA, Wilson W, Steckelberg J, Karchmer AW, et al. Diagnosis and management of infective endocarditis and its complications. Circulation. 1998;98:2936-48

6. Suknjaja V, Popovic N, Božic S, Sakalaš L, Milojkovic J, Hajder D. Embolic events and neurological complications in infective endocarditis. Curr Top Neurol Psychiatr Relat Discip. 2011;19:49-53.

7. Ducruet AF, Hickman JL, Zacharia BE, Narula R, Grobelnv BT, Gorski J, et al. Intracranial infectious aneurysms: a comprehensive review. Neurosurg Rev. 2010;33:37-46.

8. Durack DT, Lukes AS, Bright DK. New criteria for diagnosis of infective endocarditis: utilization of specific echocardiographic findings. Duke Endocarditis Service. Am J Med. 1994;96:200-9.

9. Wilson LJT, Hareedran A, Grant M, Baird T, Schulz UG, Muir KW, et al. Improving the assessment of outcomes in stroke: use off a structured interview to assign grades on the modified rankin scale. Stroke. 2002;33:2243-6.

10. Habib G, Hoen B, Tornos P, Thuny F, Prendergast B, Vilacosta I, et al. Guidelines on the prevention, diagnosis and treatment of infective endocarditis. Eur Heart J. 2009;30:2369-413.

11. Westphal N, Plicht B, Naber C. Infective endocarditis-prophylaxis, diagnostic criteria, and treatment. Dtsch Arztebl Int. 2009;106:481-9.

12. Kazelian LR, Vidal LA, Neme R, Gagliardi JA. [Endocarditis infecciosa activa: 152 casos]. Medicina (B Aires) 2012;72:109-14.
13. Pessinaba S, Kane A, Ndiaye NB, Mbaye A, Bodian M, Dina MM, et al. Vascular complications of infective endocarditis. Med Mal Infect. 2012;42:213-7.

14. Fukuda W, Daitoku K, Minakawa M, Fukui K, Suzuki Y, Fukuda I. Infective endocarditis with cerebrovascular complications: timing of surgical intervention. Interact Cardiovasc Thorac Surg. 2011;14:26-30.

15. Yeates A, Mundy J, Griffin R, Marshall L, Wood A, Peters P, et al. Early and mid-term outcomes following surgical management of infective endocarditis with associated cerebral complications: a single centre experience. Heart Lung Circ. 2010;19:523-7.

16. Romain S, Mirabel M, Hajage D, Tubach F, Vignon P, Perez P, et al. Neurologic complications and outcomes of infective endocarditis in critically ill patients: The ENDOcardite en REAnimation prospective multicenter study. Crit Care Med. 2011;39:1474-81

17. Fideli U, Schievano E, Buonfrate D, Pellizzer G, Spolaore P. Increasing incidence and mortality of infective endocarditis: a population-based study through a record-linkage system. BMC Infect Dis. 2011;11:2-7.

\section{Acronyms}

IE - Infectious endocarditis

HMSJ - Hospital Municipal São José 